


\section{El pro-ducir del diseño}

\section{The Pro-duce of design}

Ivonne Ortiz Sánchez

ISSN (imp): 1390-4825

ISSN (e): 2477-9199
Fecha de recepción: 15/10/17 Fecha de aceptación: 10/05/18

\section{Resumen:}

El producir del diseño es un texto desarrollado a partir de un estudio que se respalda en la obra de M. Heidegger. Se toman dos de sus obras relevantes: La pregunta por la técnica y Construir, habitar, pensar. E el primero, se aborda la técnica como medio para un fin en búsqueda de la esencia en el producir; en el segundo, se enfoca la aproximación a la sustancia del construir y el habitar que se dirigen hacia el producir. Esto con el fin de lograr una aproximación a la filosofía del diseño para sustentar su desarrollo en tres ámbitos fundamentales: 1) El diseño como proceso; 2) El diseño como práctica advertida por el contexto; y, 3) El diseńo como valor activo pero diferenciado a través del artefacto. Este acercamiento tiene como atributo el reconocer el carácter humanista del diseño en la contemporaneidad.

\section{Palabras clave:}

Técnica, producir, diseño, proceso de diseño, construir, habitar, diseñador

\section{Abstract:}

The Produce of Design is a text developed from a study that is supported in the work of M. Heidegger. Two of his relevant works are taken in consideration: The question about technique and Construct, to inhabit, to think; in the first one, the technique is addressed as a means to an end in search of essence in the production; in the second text, the approach to the substance of building and inhabiting that are directed towards producing. This, in order to achieve an approach to the philosophy of design to support development in three key areas: 1) Design as a process; 2) Design as a practice noticed by the context; and, 3) Design as an active but differentiated value through the artifact. This approach has the attribute of recognizing the humanistic character of design in the present.

\section{Key Words:}

Technique, produce, design, design process,build, inhabit, designer

\section{Biografía de la Autora:}

Ivonne Ortiz Sánchez, Quito 1985. Graduada en Diseńo con mención en Productos por la PUCE. Estudios en la Universidade de Lisboa en la Faculdade de Belas Artes, obteniendo el título de Mestre em Design de Equipamento com especialização em Estudos de Design. Investigadora sobre temas relacionados con el ecodiseño, la ética en el proyecto de diseño, la filosofía y teoría del diseño y la crítica profesional del diseño. Se ha hecho cargo de proyectos de diseño de productos, diseńo gráfico, diseńo de vestuario y accesorios. Es docente de la FADA-PUCE desde el 2015. 
El Diseño es una profesión y una actividad social que ha evolucionado un enfoque que buscaba satisfacer las exigencias de la producción en masa y los requerimientos de la industria, que luego privilegiaba los requerimientos del usuario, y que luego se centró tanto en el diseño de servicios como en el diseño de productos para que los proyectos sean sociales, igualitarios e inclusivos, $y$ estén orientados conscientemente hacia el factor medioambiental. De esta manera, el rol del diseñador actualmente constituye una forma de cuestionar las actitudes y los sistemas político, económico y sociocultural que se diseminan en las sociedades. De esta manera, el diseñador es capaz de generar formas de pensamiento y crítica si adopta una postura crítica para producir propuestas alternativas que pretenda crear conciencia social y cultural.

Por estas razones el universo del diseño está constituido por varias esferas: 1) El diseńo como proceso: Se define como la metodología y el proyecto de diseño. 2) El diseño como práctica informada por el contexto: Explica cómo el proceso de diseño y el artefacto están influenciados directamente por los contextos geográficos específicos de cada región o lugar. 3) El diseño como valor omnipresente pero diferenciado a través del artefacto: Se define como la conjunción de lugares, artefactos y prácticas que ayudan a crear una cultura vasta y diversa que a su vez es específica y distintiva ${ }^{1}$.

Generalmente, cuando hablamos de la profesión en el campo del diseño se piensa en creación, generación y producción; sin embargo, esto no sería posible sin la instancia reflexiva que ocurre a través de preguntas como: ¿Qué es lo que hacemos? ¿Cuál es nuestra responsabilidad al traer a presencia un objeto? ¿Estamos elaborando un necesario proceso producción? ¿Cómo lo hacemos? ¿Cuál es la esencia de los objetos que creamos? Estos cuestionamientos emergen de modo crítico en un desarrollo profesional que, evitando en lo posible el procesamiento mecánico y deshumanizante, afirme a través del diseño el carácter humanista de su producción.

Siendo así, la actividad proyectual amerita formas de pensamiento y razonamiento para el acierto

${ }^{1}$ Esta división del universo del diseño se desarrolla dentro de la investigación de tesis de maestría Responsabilidades éticas en el proyecto de Diseño, desarrollados por la autora del presente texto. de las problemáticas a enfrentar, sean de índole espacial, gráfica, objetual o de servicio social; que, luego de resolverse conceptual y descriptivamente, pasa hacia la materialización a través de la fabricación industrial.

Esta templanza, evidentemente, requiere de métodos y técnicas para determinar las cualidades del objeto, el cual tendrá como propósito cubrir una necesidad humana cotidiana o de emergencia. En primer lugar, la etnografía rápida (observar y aprender de esta fuente primaria) consiste en pasar el mayor tiempo posible con la persona que va a ser el usuario del elemento a desarrollar, ganando su confianza y dando seguridad hasta la conclusión del proceso.

En segundo lugar, a partir de estos conocimientos pasamos a una fase de investigación y análisis, que permiten obtener información para desarrollar el proyecto, a través de los requerimientos del usuario para definir la contextura del objeto, los materiales a utilizarse y el proceso de fabricación. En esta etapa se utilizan encuestas, entrevistas y mapas cognitivos para esbozar las respuestas a la problemática que se enfrenta.

En tercer lugar, obtenida la información preliminar, se inicia el proceso de creación y configuración a través de distintas técnicas, como por ejemplo: los bocetos, los modelos y las maquetas de estudio, partiendo de la conceptualización, estudio de referentes, entre otros. En cuarto lugar, se analizan las opciones observando cuál es la pertinente, esto se realiza cotejando los requerimientos previamente establecidos y, una vez confrontadas las propuestas y escogida la óptima, se pule al detalle la propuesta antes de pasar a la etapa de construcción del artefacto.

En el proceso de hacer tangible configur el artefacto, se manifiesta la materialización del diseño a través de la apariencia del objeto conseguido y su funcionalidad. Las características y significados particulares de este han dependido del enunciado de la necesidad y su contexto. De este modo se evidencia que el diseño, como actividad, y el artefacto, como objeto, configuran un mensaje que busca ser comunicado y entendido.

Heidegger en el texto La pregunta por la técnica, señala que "el preguntar abre un camino (...) el camino del pensar (...) Preguntamos por la técnica y con ello quisiéramos una relación libre con ella. 
Libre es la relación cuando abre nuestro ser-ahi (Dasein) a la esencia de la técnica” (Heidegger, 1997, p. 113). Este texto, en cierta manera, da contestación a las preguntas anteriores y, configura reflexiones en torno a la búsqueda de la esencia del diseño. Esto significa, entender el diseño para encontrar su sentido humanista, así como nuevas relaciones sociales tendientes a clarificar el camino a seguir en la contemporaneidad.

Por ello, para describir qué es y qué hace la técnica, Heidegger anota: "Una dice: La técnica es un medio para un fin; la otra dice: Técnica es un hacer del hombre" (Heidegger, 1997, p. 114). Por consiguiente, el diseño como técnica es un medio: para un procedimiento de producción y un medio para resolver una problemática que parte de una necesidad evidente; $y$, como valor omnipresente pero diferenciado a través del artefacto, permite realizar una actividad concreta. Pero también, como causa y efecto, como técnica el diseño es un hacer de la persona; es decir, es un hacer del diseñador; como proceso, es una sucesión de movimientos en que se realizan actividades específicas que permiten prefigurar el objeto a través de instrumentos y herramientas utilizados por el diseñador.

"Un medio es aquello por medio de lo cual algo es hecho, y así, obtenido. Lo que tiene por consecuencia un efecto, se llama causa" (Heidegger, 1997, p. 115) (...) "La filosofía enseña desde hace siglos que hay cuatro causas: 1. La causa materialis, el material, con la que se prepara(...); 2. La causa formalis, la forma en la que se traduce la materia; 3. La causa finalis, el fin (...); y 4. La causa efficiens, que produce el efecto" (Heidegger, 1997, p. 116)

El diseño, como valor omnipresente pero diferenciado a través del artefacto, tiene un efecto, cuyo resultado es que valor del objeto se identifica con su causa y, en sí mismo, evoca las cuatro causas propuestas en la filosofía heideggeriana. La causa material remite a la cuestión de la consistencia del objeto, la cual se concreta en el material, y permite connotar características específicas que le dan una identidad única. La causa formal del objeto, esto es su forma, es el límite que le da figura y lo contiene, y se relaciona así con la causa material que lo determina tangible. Este es el motivo que el diseńador enfoca en la causa del objeto y comunica el mensaje crítico, de función y uso, ya sea a través de sus partes o de la totalidad.

Por ello la causa final es la función misma para la que fue creado. La unión de materialidad y forma crea el vínculo de significado asociado al objeto; por tanto, la materialidad es lo que le suministra las características específicas e implícitas, que se comprenden tanto a partir del usuario como del diseñador; lo cual se denota a través de la forma del objeto logrado. Y, por último, está la causa eficiente que determina la causalidad del artefacto en el ser-ahí (Dasein); en este caso, se trata del diseñador implicado con su ser y su hacer en este proceso.

En este contexto, y para entender lo propuesto sobre las causas del artefacto, se puede pensar en el ejemplo de una tetera. Cuando esta es desde su causa material, se nos remite a la cuestión de su consistencia y de las características específicas dadas por el material. Si, por ejemplo, es de cerámica, se la percibe robusta y resistente, si es de cristal, se aprecia su transparencia.

Estas características dependen de dos motivos: por un lado, la necesidad y gusto del usuario y, por otro, la forma cómo el diseñador enfrenta la circunstancia de esta necesidad hasta obtener un objeto estéticamente permisible, acertadamente construido y apropiadamente único. Por otra parte, la tetera, desde su causa formal, nos remite a las partes que conforman el todo descrito y nos dan a conocer lo que es; es así, que la tetera tiene como resultado: un elemento contenedor, un asa que permite manipularla y una tapa que cubre el contenido. Desde su causa final, la tetera es un objeto que contiene líquidos como café o té, y lo mantiene caliente. Quien determina esta causalidad es el diseñador la produce.

Las cuatro causas son modos de ser-responsable-de, que se co-pertenecen entre sí (...) los cuatro modos de ser-responsable-de traen algo a aparecer. Le permiten pro-venir a la presencia (...). Todo dar-lugar-a que algo (cualquiera que sea) vaya y proceda desde lo no-presente a la presencia, (...); es pro-ducir (...). El dar-lugar-a atañe a la presencia de lo que aparece en el pro-ducir, en cada caso. El pro-ducir pro-duce desde el velamiento al desvelamiento. El pro-ducir acontece solamente cuando llega lo velado a lo desvelado. Este llegar se mueve y descansa en lo que nosotros 
llamamos desocultar (...). La técnica es un modo del desocultar. La técnica presencia en el ámbito en el que acontece desocultar y desvelamiento, (...), verdad. (Heidegger, 1997, p. $117-120,122)$

Cuando nos remitimos a los modos del ser-responsable-de, tenemos que diferenciar el enfoque, ya que tenemos una causa formal que da-lugar-a la creación del objeto, como contenedor; una causa material que da-lugar-a la fabricación del objeto, que se remite a su consistencia; una causa final que da-lugar-a lo que es el objeto y su funcionalidad; y una causa eficiente que es el Dasein: el diseñador que da-lugar-al objeto en sí, es decir quien, través de las causas del objeto, lo pro-viene, lo origina, lo trae a la presencia y lo produce.

Aun cuando cada causa nos muestra un modo de ser-responsable-de distinto para cada causa, estas solo pueden funcionar en conjunto y a través del diseñador. Podemos entonces afirmar que la esencia del diseño y del diseñador es el pro-ducir del artefacto, ya que a través de la técnica mediante la cual se produce el objeto, nos muestra lo que estaba oculto, lo desvela y lo trae a presencia.

Por su parte el texto de Heidegger Construir, habitar, pensar nos remite a la crítica y la reflexión sobre el sentido del construir, el cual ha sido desligado del habitar, que, en su esencia, no significa solo morar en un lugar. Es interesante notar que este texto, entre sus conclusiones, señala que el construir es tanto un cuidar proveniente del cultivar, como un custodiar. Esta observación se refiere a las cosas que, convertidas en objetos construidos, albergan o representan al hombre, pero también se refiere al el erigir, que es construir en sentido estricto. De esta manera se cuidan las cosas porque albergan la esencia de los hombres y se erigen cosas porque dan paso a los lugares del "ser".

El construir como el habitar, es decir, estar en la tierra, para la experiencia cotidiana del ser humano es desde siempre, como lo dice tan bellamente la lengua, lo "habitual" (...). El habitar es más bien siempre un residir junto a las cosas. El habitar como cuidar guarda (custodia) la Cuaternidad en aquello junto a lo cual los mortales residen: las cosas (...) El verdadero cuidar es algo positivo, y acontece cuando de antemano dejamos algo en su esencia, cuando propiamente re-albergamos algo en su esencia(...). (Heidegger, 1951, p. $2,3$ y 4$)$

Cuando Heidegger habla del cuidar, es decir proteger las cosas que representan a los hombres, da cuenta de aquello que las trae a su esencia, lo cual se corresponde con la idea de que la esencia del diseño es producir el artefacto a través de sus cuatro causas. Proteger los objetos es traerlos a su esencia, que es producirlos. En este sentido, el pro-ducir el objeto da paso al lugar ya que permite albergar dentro de este a la cuaternidad. Este tipo de pro-ducir objetos que dan lugares es el construir y este construir es el habitar. De este modo el habitar es, producir.

Así, concluimos que la esencia del diseño es pro-ducir el artefacto, es decir, traerlo a la presencia. Esto a su vez permite pro-ducir lugares a través del objeto, que es la esencia del diseño, y, que de ese modo, alberga la esencia de los hombres. En resumen, esta manera de pro-ducir significa construir y habitar, lo cual que es la esencia de lo que hace el hombre.

\section{BIBLIOGRAFÍA}

Heidegger, M. (1951). Construir, habitar, pensar. Recuperado de: http://www.lugaradudas. org/archivo/publicaciones/fotocopioteca/39 heidegger.pdf

Heidegger, M. (1997). Filosofía, Ciencia y Técnica. Santiago de Chile, Editorial Universitaria.

Ortiz, Ivonne. (2014). Responsabilidades éticas en el proyecto de Design. Tesis de maestría: Universidade de Lisboa, Faculdade de Belas Artes, Lisboa.

Calvera, Anna. (2007). El cosear de las cosas. Consideraciones rezagadas a partir de Martin Heidegger. En Calvera, Anna. (Ed.), De lo bello de las cosas. Materiales para una estética del diseño (101-123). Barcelona: Editorial Gustavo Gili. Abalos, Iñaki (2000). La buena vida: visita guiada a las casas de la modernidad. Barcelona: Editorial Gustavo Gili. 
INDEX \#05 